\title{
APPROXIMATION OF FIXED POINTS OF STRONGLY PSEUDOCONTRACTIVE MAPPINGS
}

\author{
C. E. CHIDUME
}

(Communicated by Palle E. T. Jorgensen)

\begin{abstract}
Let $E$ be a real Banach space with a uniformly convex dual, and let $K$ be a nonempty closed convex and bounded subset of $E$. Let $T: K \rightarrow K$ be a continuous strongly pseudocontractive mapping of $K$ into itself. Let $\left\{c_{n}\right\}_{n=1}^{\infty}$ be a real sequence satisfying: (i) $0<c_{n}<1$ for all $n \geq 1$; (ii) $\sum_{n=1}^{\infty} c_{n}=\infty$; and (iii) $\sum_{n=1}^{\infty} c_{n} b\left(c_{n}\right)<\infty$, where $b:[0, \infty) \rightarrow[0, \infty)$ is some continuous nondecreasing function satisfying $b(0)=0, b(c t) \leq c b(t)$ for all $c \geq 1$. Then the sequence $\left\{x_{n}\right\}_{n=1}^{\infty}$ generated by $x_{1} \in K$,

$$
x_{n+1}=\left(1-c_{n}\right) x_{n}+c_{n} T x_{n}, \quad n \geq 1,
$$

converges strongly to the unique fixed point of $T$. A related result deals with the Ishikawa iteration scheme when $T$ is Lipschitzian and strongly pseudocontractive.
\end{abstract}

\section{INTRODUCTION}

Let $E$ be a normed linear space, $K \subseteq E$. A mapping $T: K \rightarrow K$ is called a strong pseudocontraction if there exists $t>1$ such that the inequality

$$
\|x-y\| \leq\|(1+r)(x-y)-r t(T x-T y)\|
$$

holds for all $x, y$ in $K$ and $r>0$. If $t=1$ then $T$ is called pseudocontractive. Interest in pseudocontractive mappings stems mainly from their connection with the important class of nonlinear accretive operators. A mapping $U$ with domain $\mathrm{D}(U)$ and range $\mathrm{R}(U)$ in $E$ is called accretive [5] if the inequality

$$
\|x-y\| \leq\|x-y+s(U x-U y)\|
$$

holds for every $x, y \in \mathrm{D}(U)$ and for all $s \geq 0$. The firm connection between the pseudocontractive and accretive mappings is that a mapping $T$ is pseudocontractive if and only if $(I-T)$ is accretive [5, Proposition 1]. Consequently, the mapping theory for accretive operators is closely related to the fixed point theory of pseudocontractive mappings.

The accretive operators were introduced independently in 1967 by Browder [5] and Kato [15]. An early fundamental result in the theory of accretive operators, due to Browder, states that the initial value problem

$$
\frac{d u}{d t}+T u=0, \quad u(0)=u_{0}
$$

Received by the editors January 21, 1992 and, in revised form, June 3, 1992.

1991 Mathematics Subject Classification. Primary 47H17. 
is solvable if $T$ is locally Lipschitzian and accretive in $E-$ a result which was subsequently generalized by Martin [20] to the continuous accretive operators. For more on the applications of accretive operators the reader may consult [4, 5].

Pseudocontractive operators have been studied by various authors (see, e.g., $[2,6-8,10,12,13,16,17,21,22])$. In [2] Bogin studied the connection between strong pseudocontraction mappings and strongly accretive mappings (defined below). He proved that $U$ is a strong pseudocontraction if and only if $(I-U)$ is a strong accretive operator. Furthermore, he proved a fixed point theorem in Banach spaces for Lipschitz strong pseudocontractions and, as a consequence, obtained a mapping theorem of Browder for this class of operators.

In [7] the author studied iterative methods for approximating fixed points of Lipschitz strongly pseudocontractive operators and proved that, if $E=L_{p}$ (or $\left.l_{p}\right), p \geq 2, K \subseteq E$, and $T: K \rightarrow K$ is a Lipschitz strongly pseudocontractive map with a nonempty fixed point set, an iteration method of the type introduced by Mann [19] converges strongly to a fixed point of $T$. The method of [7] also gives that such a fixed point is necessarily unique. However, the method could not be adapted either to prove the above results when $E=L_{p}$ (or $l_{p}$ ), $1<p \leq 2$, or to establish whether or not another well-known fixed point iteration method, the Ishikawa iteration method (see, e.g., [13]) converges to the fixed point of $T$.

It is our purpose in this paper to resolve these questions by proving the following much more general result: If $E$ is any real Banach space with a uniformly convex dual space $E^{*}, K \subseteq E$, and $T: K \rightarrow K$ is any strongly pseudocontractive map with a nonempty fixed point set, then both the Mann iteration method and the Ishikawa iteration method converge strongly to the unique fixed point of $T$. In particular, our Theorem 1 extends the result of [7] to the larger class of continuous strongly pseudocontractive maps and from $L_{p}$ spaces $(p \geq 2)$ to the more general Banach spaces considered here. (See also our comments following the proof of Theorem 2.) Furthermore, explicit error estimates are also given.

Remark 1. The Ishikawa iteration method was introduced in [13] to approximate a fixed point of any Lipschitz pseudocontractive map $U$ in a Hilbert space when the domain of the map is also assumed to be convex and compact. It is still an open question whether or not the Mann iteration process converges to a fixed point of $U$ (see, e.g., [12, p. 504]).

\section{Preliminaries}

We start by defining the two fixed point iteration methods which will be needed in the sequel.

A. The Ishikawa Iteration Method (see, e.g., [13, 28]). If $K$ is a convex subset of a real Banach space $E$ and $T$ is a mapping of $K$ into itself, the sequence $\left\{x_{n}\right\}_{n=0}^{\infty}$ in $K$ is defined by

$$
\begin{gathered}
x_{0} \in K, \\
x_{n+1}=\left(1-\lambda_{n}\right) x_{n}+\lambda_{n} T y_{n}, \\
y_{n}=\left(1-\beta_{n}\right) x_{n}+\beta_{n} T x_{n}, \quad n \geq 0,
\end{gathered}
$$


where $\left\{\lambda_{n}\right\}_{n=0}^{\infty},\left\{\beta_{n}\right\}_{n=0}^{\infty}$ satisfy $0 \leq \lambda_{n} \leq \beta_{n}<1$ for all $n ; \lim _{n \rightarrow \infty} \beta_{n}=0$, and $\sum_{n=0}^{\infty} \lambda_{n} \beta_{n}=\infty$.

B. The Mann Iteration Method (see, e.g., $[19,28]$ ). With $E, K$, and $x_{0}$ as in A, the sequence $\left\{x_{n}\right\}_{n=0}^{\infty}$ in $K$ is defined by

$$
x_{n+1}=\left(1-c_{n}\right) x_{n}+c_{n} T x_{n}, \quad n \geq 0,
$$

where $\left\{c_{n}\right\}_{n=0}^{\infty}$ is a real sequence satisfying $c_{0}=1,0 \leq c_{n}<1$ for all $n \geq 1$, and $\sum_{n=0}^{\infty} c_{n}=\infty$. The condition $\sum_{n=0}^{\infty} c_{n}=\infty$ is, in some applications, replaced by $\sum_{n=0}^{\infty} c_{n}\left(1-c_{n}\right)=\infty$.

The iteration methods $A$ and $B$ have successfully been employed by various authors to approximate solutions of nonlinear operators in Banach spaces (see, e.g., [6-8, 11-14, 18, 19, 22-28]). For a detailed comparison of the two methods for various classes of nonlinear operators the reader may consult [28].

For a Banach space $E$ we shall denote by $J$ the duality map from $E$ to $2^{E^{*}}$ defined by

$$
J x=\left\{f^{*} \in E^{*}:\left\|f^{*}\right\|^{2}=\|x\|^{2}=\left\langle x, f^{*}\right\rangle\right\},
$$

where $E^{*}$ denotes the dual space of $E$ and $\langle\cdot, \cdot\rangle$ denotes the generalized duality pairing. It is known that, if $E^{*}$ is uniformly convex, then $J$ is singlevalued. In the sequel we shall denote the single-valued normalized duality map by $j$. Thus, by a single-valued normalized duality map, we shall mean a map $j: E \rightarrow E^{*}$ such that, for each $u \in E, j(u)$ is an element of $E^{*}$ which satisfies the conditions

$$
\langle u, j(u)\rangle=\|u\| \cdot\|j(u)\|, \quad\|j(u)\|=\|u\| .
$$

We remark immediately that the accretive condition (2) can be expressed in terms of the duality map as follows (see, e.g., [15]): For each $x, y \in D(U)$, there exists some $\omega \in J(x-y)$ such that

$$
\operatorname{Re}\langle U x-U y, \omega\rangle \geq 0 \text {. }
$$

Now let $K \subseteq E$. A mapping $A: K \rightarrow K$ is called strongly accretive if for each $x, y \in K$ there exists $\omega \in J(x-y)$ such that

$$
\langle A x-A y, \omega\rangle \geq k\|x-y\|^{2}
$$

for some constant $k>0$. Without loss of generality we shall assume that $k \in(0,1)$.

In the sequel we shall also need the following remarks.

Remark 2. In [24, p. 89] Reich proved that if $E^{*}$ is uniformly convex then there exists a continuous nondecreasing function $b:[0, \infty) \rightarrow[0, \infty)$ such that $b(0)=0, b(c t) \leq c b(t)$ for all $c \geq 1$, and

$$
\|x+y\|^{2} \leq\|x\|^{2}+2\langle y, j(x)\rangle+\max \{\|x\|, 1\}\|y\| b(\|y\|)
$$

for all $x, y \in E$.

Remark 3. Nevanlinna and Reich [23] showed that, for any given continuous nondecreasing function $b(t)$ with $b(0)=0$, sequences $\left\{\lambda_{n}\right\}_{n=0}^{\infty}$ always exist satisfying:

(i) $0<\lambda_{n}<1$ for all $n \geq 0$,

(ii) $\sum_{n=0}^{\infty} \lambda_{n}=\infty$, and

(iii) $\sum_{n=0}^{\infty} \lambda_{n} b\left(\lambda_{n}\right)<\infty$. 
For $E=L_{p}, 1<p<\infty$, we can choose any sequence $\left\{\lambda_{n}\right\}_{n=0}^{\infty}$ in $l^{s} / l^{1}$ with $s=p$ if $1<p \leq 2$ and $s=2$ if $p \geq 2$.

In the remainder of this paper, the Lipschitz constant of $T$ will be denoted by $L(\geq 1)$.

\section{MAIN Results}

We prove the following theorems.

Theorem 1. Let $E$ be a real Banach space with a uniformly convex dual, $E^{*}$, and let $K$ be a nonempty closed convex and bounded subset of $E$. Let $T: K \rightarrow K$ be a continuous strongly pseudocontractive mapping of $K$ into itself. Let $\left\{c_{n}\right\}_{n=1}^{\infty}$ be a real sequence satisfying.

(i) $0<c_{n}<1$ for all $n \geq 1$,

(ii) $\sum_{n=1}^{\infty} c_{n}=\infty$, and

(iii) $\sum_{n=1}^{\infty} c_{n} b\left(c_{n}\right)<\infty$.

Then the sequence $\left\{x_{n}\right\}_{n=1}^{\infty}$ generated by $x_{1} \in K$,

$$
x_{n+1}=\left(1-c_{n}\right) x_{n}+c_{n} T x_{n}, \quad n \geq 1,
$$

converges strongly to the unique fixed point of $T$.

Proof. The existence of a fixed point follows from Deimling [9]. Let $x^{*}$ denote a fixed point of $T$. Since $T$ is strongly pseudocontractive, $(I-T)$ is strongly accretive and, for each $x, y \in K$,

$$
\langle(I-T) x-(I-T) y, j(x-y)\rangle \geq k\|x-y\|^{2},
$$

where $k=(t-1) t^{-1}$ (see, e.g., $\left.[2,7]\right)$. Using (6)-(8) we have

$$
\begin{aligned}
\| x_{n+1} & -x^{*}\left\|^{2}=\right\|\left(1-c_{n}\right)\left(x_{n}-x^{*}\right)+c_{n}\left(T x_{n}-T x^{*}\right) \|^{2} \\
\leq & \left(1-c_{n}\right)^{2}\left\|x_{n}-x^{*}\right\|^{2}+2 c_{n}\left(1-c_{n}\right)\left\langle T x_{n}-T x^{*}, j\left(x_{n}-x^{*}\right)\right\rangle \\
& +\max \left\{\left(1-c_{n}\right)\left\|x_{n}-x^{*}\right\|, 1\right\} c_{n}\left\|T x_{n}-T x^{*}\right\| b\left(c_{n}\left\|T x_{n}-T x^{*}\right\|\right) \\
\leq & \left(1-c_{n}\right)^{2}\left\|x_{n}-x^{*}\right\|^{2}+2(1-k) c_{n}\left(1-c_{n}\right)\left\|x_{n}-x^{*}\right\|^{2} \\
& +\max \left\{\left(1-c_{n}\right)\left\|x_{n}-x^{*}\right\|, 1\right\} c_{n}\left\|T x_{n}-T x^{*}\right\| \max \left\{\left\|T x_{n}-T x^{*}\right\|, 1\right\} b\left(c_{n}\right) \\
\leq & {\left[\left(1-c_{n}\right)^{2}+2(1-k) c_{n}\left(1-c_{n}\right)+(1-k)^{2} c_{n}^{2}\right]\left\|x_{n}-x^{*}\right\|^{2}+M c_{n} b\left(c_{n}\right), }
\end{aligned}
$$

for some constant $M>0$, since $K$ is bounded. Thus,

$$
\left\|x_{n+1}-x^{*}\right\|^{2} \leq\left(1-k c_{n}\right)\left\|x_{n}-x^{*}\right\|^{2}+M c_{n} b\left(c_{n}\right) \text {. }
$$

Set $\rho_{n}=\left\|x_{n}-x^{*}\right\|^{2}, \gamma_{n}=k c_{n}$, and $\delta_{n}^{2}=M c_{n} b\left(c_{n}\right)$ to obtain $\rho_{n+1} \leq$ $\left(1-\gamma_{n}\right) \rho_{n}+\delta_{n}^{2}$, which is inequality (10) of [7] with $\delta_{n}^{2}=c_{n}^{2} d^{2}$. The rest of the argument now follows as in [7] to give that $\left\{x_{n}\right\}_{n=0}^{\infty}$ converges strongly to the unique fixed point of $T$.

Remark 4. As was mentioned in the introduction, Theorem 1 extends the Theorem of [7] to the much larger class of continuous strongly pseudocontractive mappings and from $L_{p}$ spaces $(p \geq 2)$ to the more general Banach spaces $E$ considered here.

If $E=L_{p}, 1<p<\infty$, Theorem 1 can be stated more simply as follows: 
Corollary 1. Let $E=L_{p}$ (or $\left.l_{p}\right), 1<p \leq 2$, and $K$ and $T$ be as in Theorem 1. Let $\left\{c_{n}\right\}_{n=1}^{\infty}$ be a real sequence satisfying.

(i) $0<c_{n}<1$ for all $n \geq 1$,

(ii) $\sum_{n=1}^{\infty} c_{n}=\infty$, and

(iii) $\sum_{n=1}^{\infty} c_{n}^{p}<\infty$.

Then the sequence $\left\{x_{n}\right\}_{n=1}^{\infty}$ generated by $x_{1} \in K$,

$$
x_{n+1}=\left(1-c_{n}\right) x_{n}+c_{n} T x_{n}, \quad n \geq 1,
$$

converges strongly to the unique fixed point of $T$.

Proof. Remark 3 and conditions (i) and (iii) imply $\sum_{n=0}^{\infty} c_{n} b\left(c_{n}\right)<\infty$. The result then follows from Theorem 1 .

Corollary 2. Let $E=L_{p}$ (or $\left.l_{p}\right), 2 \leq p<\infty$, and let $K$ and $T$ be as in Theorem 1. Let $\left\{c_{n}\right\}_{n=1}^{\infty}$ be a real sequence satisfying.

(i) $0<c_{n}<1$ for all $n \geq 1$,

(ii) $\sum_{n=1}^{\infty} c_{n}=\infty$, and

(iii) $\sum_{n=1}^{\infty} c_{n}^{2}<\infty$.

Then the sequence $\left\{x_{n}\right\}_{n=1}^{\infty}$ generated by $x_{1} \in K$,

$$
x_{n+1}=\left(1-c_{n}\right) x_{n}+c_{n} T x_{n}, \quad n \geq 1,
$$

converges strongly to the unique fixed point in $T$.

Proof. Follows as in the proof of Corollary 1.

Error estimates. Following the method of [8] and setting $c_{n}=s(n+1)^{-1}$ we obtain that the error estimate in Theorem 1 is given by

$$
\left\|x_{n}-x^{*}\right\|=O\left(n^{(-(s-1)) / 2}\right) \text {. }
$$

If $E=L_{p}\left(\right.$ or $\left.l_{p}\right)$ then

$$
\left\|x_{n}-x^{*}\right\|=O\left(n^{-(p-1) / 2}\right) \text { if } 1<p \leq 2
$$

and

$$
\left\|x_{n}-x^{*}\right\|=O\left(n^{-1 / 2}\right) \text { if } p \geq 2 .
$$

Theorem 2. Let $E$ be a real Banach space with a uniformly convex dual space, $E^{*}$, and let $K$ be a nonempty closed convex and bounded subset of $E$. Let $T: K \rightarrow K$ be a Lipschitz strongly pseudocontractive mapping of $K$ into itself. Let $\left\{\alpha_{n}\right\}_{n=1}^{\infty}$ and $\left\{\beta_{n}\right\}_{n=1}^{\infty}$ be real sequences satisfying.

(i) $0 \leq \alpha_{n} \leq \beta_{n}<1$ for all $n \geq 1$,

(ii) $\sum_{n=1}^{\infty} \alpha_{n}=\infty$,

(iii) $\lim _{n} \beta_{n}=0$, and

(iv) $\sum_{n=1}^{\infty} \alpha_{n} b\left(\alpha_{n}\right)<\infty$.

For arbitrary $x_{1} \in K$, define the sequence $\left\{x_{n}\right\}_{n=1}^{\infty}$ in $K$ by

$$
\begin{gathered}
x_{n+1}=\left(1-\alpha_{n}\right) x_{n}+\alpha_{n} T y_{n}, \\
y_{n}=\left(1-\beta_{n}\right) x_{n}+\beta_{n} T x_{n}, \quad n \geq 1 .
\end{gathered}
$$

Then $\left\{x_{n}\right\}_{n=1}^{\infty}$ converges strongly to the unique fuxed point of $T$. 
Proof. The existence of a fixed point follows from Deimling [9]. Let $x^{*}$ denote a fixed point of $T$. Using (6) and (8)-(10) we obtain

$$
\begin{aligned}
\left\|x_{n+1}-x^{*}\right\|^{2}= & \left\|\left(1-\alpha_{n}\right)\left(x_{n}-x^{*}\right)+\alpha_{n}\left(T y_{n}-T x^{*}\right)\right\|^{2} \\
\leq & \left(1-\alpha_{n}\right)^{2} \|\left(x_{n}-x^{*} \|^{2}+2 \alpha_{n}\left(1-\alpha_{n}\right)\left\langle T y_{n}-T x^{*}, j\left(x_{n}-x^{*}\right)\right\rangle\right. \\
& +\max \left\{\left(1-\alpha_{n}\right)\left\|x_{n}-x^{*}\right\|, 1\right\} \\
& \cdot \alpha_{n}\left\|T y_{n}-T x^{*}\right\| \max \left\{\left\|T y_{n}-T x^{*}\right\|, 1\right\} b\left(\alpha_{n}\right),
\end{aligned}
$$

and, using triangle inequality and (8),

$$
\begin{aligned}
\left\langle T y_{n}\right. & \left.-T x^{*}, j\left(x_{n}-x^{*}\right)\right\rangle \\
& =\left\langle T y_{n}-T x_{n}, j\left(x_{n}-x^{*}\right)\right\rangle+\left\langle T x_{n}-T x^{*}, j\left(x_{n}-x^{*}\right)\right\rangle \\
& \leq L\left\|y_{n}-x_{n}\right\| \cdot\left\|x_{n}-x^{*}\right\|+(1-k)\left\|x_{n}-x^{*}\right\|^{2} \\
& \leq\left\{L(1+L) \beta_{n}+(1-k)\right\}\left\|x_{n}-x^{*}\right\|^{2} .
\end{aligned}
$$

For sufficiently large $n$, condition (iii) implies $L(1+L) \beta_{n}<k^{2}$. Hence,

$$
\left\langle T y_{n}-T x^{*}, j\left(x_{n}-x^{*}\right)\right\rangle \leq[1-k(1-k)]\left\|x_{n}-x^{*}\right\|^{2}
$$

so that

$$
\left\|x_{n+1}-x^{*}\right\|^{2} \leq\left[\left(1-\alpha_{n}\right)^{2}+2 \alpha_{n}\left(1-\alpha_{n}\right)[1-k(1-k)]\right]\left\|x_{n}-x^{*}\right\|^{2}+M \alpha_{n} b\left(\alpha_{n}\right),
$$

for some constant $M>0$, since $K$ is bounded. Adding [1-k(1-k)] $]^{2}$. $\left\|x_{n}-x^{*}\right\|^{2} \alpha_{n}^{2}$ to the right side of the last inequality we obtain

$$
\left\|x_{n+1}-x^{*}\right\|^{2} \leq\left\{\left(1-\alpha_{n}\right)+\alpha_{n}[1-k(1-k)]\right\}^{2}\left\|x_{n}-x^{*}\right\|^{2}+M \alpha_{n} b\left(\alpha_{n}\right)
$$

so that $\left\|x_{n+1}-x^{*}\right\| \leq\left(1-k(1-k) \alpha_{n}\right)\left\|x_{n}-x^{*}\right\|^{2}+M \alpha_{n} b\left(\alpha_{n}\right)$. The rest of the argument now follows as in the proof of Theorem 1.

Remark 5. The error estimate for Theorem 2 is of the same order as that obtained in Theorem 1. It follows from Theorems 1 and 2 that if $K$ is a nonempty closed convex and bounded subset of a real Banach space with a uniformly convex dual and $T: K \rightarrow K$ is a Lipschitz strongly pseudocontractive map of $K$ into itself then either the Mann iteration method of the Ishikawa iteration method can be used to approximate the fixed point of $T$. However, since the error estimate with the two methods are of the same order, Mann process may be preferred due to its simplicity. Furthermore, the Mann process can be applied when $T$ is continuous and strongly pseudocontractive. It is not known whether or not the Ishikawa iteration method converges for this class of nonlinear maps.

\section{ACKNOWLEDGMENTS}

The author thanks Professor Abdus Salam, the International Atomic Energy Agency, and UNESCO for hospitality at the International Centre for Theoretical Physics, Trieste.

\section{REFERENCES}

1. N. A. Assad and W. A. Kirk, Fixed point theorems for set-valued mappings of contractive type, Pacific J. Math. 43 (1972), 553-562.

2. J. Bogin, On strict pseudo-contractions and a fixed point theorem, Technion Preprint Series No. MT-219, Haifa, Israel, 1974.

3. F. E. Browder, The solvability of nonlinear functional equations, Duke Math. J. 30 (1963), $557-566$. 
4. U.S.A. 61 (1968), 388-393.

5. __ Nonlinear mappings of nonexpansive and accretive type in Banach spaces, Bull. Amer. Math. Soc. 73 (1967), 875-882.

6. F. E. Browder and W. V. Petryshyn, Construction of fixed points of nonlinear mappings in Hilbert space, J. Math. Anal. Appl. 20 (1967), 197-228.

7. C. E. Chidume, Iterative approximation of fixed points of Lipschitzian strictly pseudocontractive mappings, Proc. Amer. Math. Soc. 99 (1987); 283-288.

8. Iterative solution of nonlinear equations of the monotone type in Banach spaces, Bull. Austral. Math. Soc. 42 (1990), 21-31.

9. K. Deimling, Zeros of accretive operators, Manuscripta Math. 13 (1974), 283-288.

10. J. A. Gatica and W. A. Kirk, Fixed point theorems for Lipschitzian pseudo-contractive mappings, Proc. Amer. Math. Soc. 36 (1972), 111-115.

11. J. Gwinner, On the convergence of some iteration processes in uniformly convex Banach spaces, Proc. Amer. Math. Soc. 81 (1978), 29-35.

12. T. L. Hicks and J. R. Kubicek, On the Mann iteration process in Hilbert space, J. Math. Anal. Appl. 59 (1977), 498-504.

13. S. Ishikawa, Fixed points by a new iteration method, Proc. Amer. Math. Soc. 149 (1944), 147-150.

14. _ Fixed points and iteration of a nonexpansive mappings in a Banach space, Proc. Amer. Math. Soc. 73 (1976), 65-71.

15. T. Kato, Nonlinear semigroups and evolution equations, J. Math. Soc. Japan 19 (1967), 508-520.

16. W. A. Kirk, A fixed point theorem for iocal pseudo-contraction in uniformly convex spaces, Manuscripta Math. 30 (1979), 89-102.

17. _ Remarks on pseudocontractive mappings, Proc. Amer. Math. Soc. 25 (1970), 820-823.

18. W. A. Kirk and C. Morales, On the approximation of fixed points of locally nonexpansive mappings, Canad. Math. Bull. 24 (1981), 441-445.

19. W. R. Mann, Mean value methods in iteration, Proc. Amer. Math. Soc. 4 (1953), 506-510.

20. R. H. Martin, Jr., A global existence theorem for autonomous differential equations in Banach spaces, Proc. Amer. Math. Soc. 26 (1970), 307-314.

21. C. Morales, Surjectivity theorems for multi-valued mappings of accretive type, Comment. Math. Univ. Carolin. 26 (1985).

22. R. N. Mukerjee, Construction of fixed points of strictly pseudocontractive mappings in generalized Hilbert spaces and related applications, Indian J. Pure Appl. Math. 15 (1966), 276-284.

23. G. Nevanlinna and S. Reich, Strong convergence of contraction semigroups and of iterative methods for accretive operators in Banach spaces, Israel J. Math. 32 (1979), 44-58.

24. S. Reich, An iterative procedure for constructing zeros of accretive sets in Banach spaces, Nonlinear Anal. 2 (1978), 85-92.

25. __ Constructing zeros of accretive operators. II, Appl. Anal. 9 (1979), 159-163.

26. Constructive techniques for accretive and monotone operators, Applied Nonlinear Analysis (V. Lakshmikantham, ed.), Academic Press, New York, 1979, pp. 335-345.

27. Math. Anal. Appl. 85 (1980), 287-292.

28. B. E. Rhoades, Comments on two fixed point iteration methods, J. Math. Anal. Appl. 56 (1976), 741-750.

International Centre for Theoretical Physics, Trieste, Italy

Permanent address: Department of Mathematics, University of Nigeria, Nsukka, Nigeria

E-mail address: chidume@ictp.trieste.it 